\title{
Heilpflanzen als Mittel gegen die Pest im Mittelalter und in der frühen Neuzeit*
}

\author{
Ellen Wulfers \\ Fislisbach (AG), Schweiz
}

\section{Schlüsselwörter}

Kräuteranwendungen · Pest · Schwarzer Tod · Mittelalter · Yersinia pestis

\section{Zusammenfassung}

Die spätmittelalterlichen Seuchenzüge der Pest in Mitteleuropa hatten einschneidende demographische und gesellschaftliche Auswirkungen. Im mittelalterlichen Krankheitsverständnis wurden verschiedene Ursachen für die Pest in Erwägung gezogen. An erster Stelle standen oft religiöse Vorstellungen; daneben spielten aber auch Überlegungen zu astrologischen Konstellationen, das Wetter, Naturereignisse, der vom Abfall in den Strassen verursachte Gestank, schlechte Ausdünstungen oder die Beulen von erkrankten Menschen eine Rolle. Die Symptomatik und Folgen der Pesterkrankung waren bestens bekannt und entsprechend gefürchtet. Aus dem damaligen Verständnis wurden verschiedenste Praktiken und Empfehlungen zum Schutz vor der Ansteckung, aber auch zur Linderung der Symptome entwickelt. Erst mit der Entdeckung des Krankheitserregers Yersinia pestis im Jahr 1894 konnten die Zusammenhänge mit den hygienischen Verhältnissen sowie der Übertragungsweg über Flöhe und häusliche Nagetiere (hauptsächlich Ratten) schlüssig nachvollzogen werden. Zwar besteht in Mitteleuropa heutzutage praktisch keine Pestgefahr mehr, doch ist das Bakterium nicht ausgerottet, sondern kommt in Afrika, Asien sowie Süd- und Mittelamerika weiterhin vor. Alte Kräuterbücher erwähnen eine Vielfalt an Pestpflanzen, die zum Schutz vor der Ansteckung, wie z.B. Gemeiner Wacholder (Juniperus communis), Kleine Bibernelle (Pimpinella saxifraga) oder Arznei-Engelwurz (Angelica archangelica), aber auch zur Behandlung der Pestbeulen dienten, wie z.B. die Schwarze Tollkirsche (Atropa belladonna). Dabei spielen innere und äussere Anwendungen (gebranntes Wasser, Salz, Pillen und Pulver bzw. Umschläge, Salben oder Pflaster) sowie Amulette, Räucherrituale oder Kräuterbüschel eine wichtige Rolle.

*Überarbeitete und aktualisierte Version einer Projektarbeit im Rahmen des CAS «Ethnobotanik und Ethnomedizin» an der Universität Zürich, Schweiz.

\section{Keywords}

Herbal therapies · Plague · Black Death · Middle Ages · Yersinia pestis

\section{Summary}

Herbal Therapies as a Means against the Plague in the Middle Ages and in early Modern Times

The plague epidemic in the late Middle Ages had a drastic impact on the demographic and societal development in Europe. The medieval perception of the plague considered several causes for the disease, many of them in a religious context. Other causes were presumed based on astrological constellations, weather conditions, natural phenomena or an act of God, or even the fetid odor caused by rubbish, vapors, or pestilential bubo. Signs, symptoms, and consequences of the plague were known and therefore greatly feared. In this historical context, different solutions and recommendations were developed in order to prevent an infection with the plague but also to ease its symptoms. Only when the bacterium Yersinia pestis was discovered in 1894, the plague epidemic could be understood in connection with unsanitary conditions and transmission routes by means of fleas and rodents (especially rats). Even though the danger of a plague epidemic in Europe is almost nonexistent nowadays, the bacterium is still virulent in Africa, Asia, as well as South and Central America. Historical herbal books document a variety of apotropaic herbs against the plague, e.g. common juniper (Juniperus communis), burnet (Pimpinella saxifraga), and angelica (Angelica archangelica), as well as herbs to treat pestilential bubo (e.g. deadly nightshade (Atropa belladonna L.)). Oral and topical applications of herbs were also documented in combination with amulets, ritual fumigations, and bunches of herbs.

\section{KARGER}

Fax +497614520714 Information@Karger.com www.karger.com 


\section{Mots-clés}

Usages des plantes médicinales · Peste · Mort noire · Moyen Âge . Yersinia pestis

\section{Résumé}

Les plantes médicinales comme médicaments contre la peste au Moyen Âge et au début des Temps modernes

Les épidémies de peste au Moyen-Âge en Europe centrale ont eu des effets démographiques et sociaux fondamentaux. Dans le contexte des connaissances de la maladie au Moyen-Âge, différents facteurs ont été considérés comme à l'origine de la peste. Les idées religieuses ont souvent occupé une place prépondérante. Mais les réflexions concernant les constellations astrologiques, la météo, les événements naturels, la puanteur causée dans les rues par les déchets, de mauvaises odeurs ou les bubons des malades ont aussi joué un rôle. La symptomatique et les séquelles de la peste étaient très bien connues et redoutées en conséquence. Issues des conceptions de l'époque, les pratiques et recommandations les plus diverses ont été développées afin de se protéger contre la contagion, mais aussi de soulager les symptômes. Ce n'est qu'en 1894 que la découverte de l'agent pathogène de la maladie, Yersinia pestis, a permis de comprendre de manière pertinente les relations de la maladie avec les conditions d'hygiène et les voies de transmission par les poux et les rongeurs domestiques (principalement les rats). Bien que le risque de peste soit pratiquement inexistant en Europe centrale, la bactérie n'est pas exterminée, mais continue d'apparaître en Afrique, Asie ainsi qu'en Amérique centrale et du Sud. Des herbiers anciens mentionnent la diversité des plantes agissant contre la peste, qui ont servi à protéger de la contagion, par ex. le genévrier commun (Juniperus communis), le petit boucage (Pimpinella saxifraga) ou I'angélique officinale (Angelica archangelica), mais aussi de traiter les bubons de la peste, comme la belladone (Atropa belladonna). Les applications internes et externes (eau-de-vie, sel, pilules et poudre ou compresses, pommades et pansements) ainsi que les amulettes, les rituels de fumage ou les touffes d'herbes y jouaient un rôle important.

\section{Einleitung}

Historische Kräuterbücher fassen unter der Bezeichnung «Pestpflanzen» Heilpflanzen zusammen, die in unterschiedlichen Zubereitungen zur Abwehr und/oder Behandlung der Pest eingesetzt wurden. Dass die Pest als epidemische Krankheit im Mittelalter eine Lebensbedrohung darstellte, zeigt die Umschreibung der Seuche als der «Schwarze Tod». Diese Bezeichnungen rufen bis heute Unbehagen hervor und werden mit weiteren Begriffen wie «Seuchenkrankheit», «Beulen», «Ungeziefer», «Gestank», «Ratten» und «Entvölkerung» assoziiert. Zur Zeit der grossen Pestepidemien in Europa waren die Symptomatik und der Krankheitsverlauf bekannt, nicht jedoch die Zusammenhänge der Krankheitsentstehung.

Die Pest hat im Mittelalter sehr viele Todesopfer gefordert. Man spricht von einem Drittel der europäischen Bevölkerung. Durch das «Wüten des Schwarzen Todes» erlitten nicht nur Einzelpersonen oder ganze Familien psychische, physische und seelische Schmerzen, vielmehr wurde die gesamte Gesellschaft dadurch beherrscht. Aufgrund dieser Pandemie entstanden deutliche Veränderungen innerhalb der Gesellschaft. Soziale Systeme funktionierten nicht mehr. Die Angst, vom Mitmenschen angesteckt zu werden, liess Menschen in elendigem $\mathrm{Zu}$ stand vereinsamen und sterben. Familiensysteme fielen auseinander. Das Kollektiv, die Gemeinsamkeit innerhalb des sozialen Systems, machte Platz für die individuelle Erfahrung und Egoismus. Antisemitismus war stark verbreitet, wurde doch behauptet, die Juden hätten die Brunnen mit der Pest kontaminiert. Dies führte an verschiedenen Orten zur Judenverfolgung und -vernichtung [1, S. 189].

Zudem änderte sich die Wirtschaftsstruktur. Arbeiter aus tieferen sozialen Schichten hatten unerwartet Zugang zu Handwerkerkreisen, da aufgrund der hohen Sterbezahl in der Bevölkerung ein grosser Mangel an Arbeitskräften vorhanden war. Dies bedeutete für die Betroffenen einen sozialen Aufstieg mit besseren finanziellen Perspektiven.

Die mittelalterliche Gesundheitspolitik war, aus unserer heutigen Sicht mit den Kenntnissen über die Bakteriologie, unzureichend. Doch gab es dazumal schon Ansätze der Regierungen und einzelner Personen, Empfehlungen abzugeben, die sehr an die heutigen Informationen zur Vermeidung einer Pandemie erinnern. Durch persönliche Erfahrungen war ersichtlich geworden, dass diese Krankheit eventuell von Mensch zu Mensch übertragbar sein könnte. So war es im italienischen Pistoia verboten, mit gebrauchten Kleidern Handel zu treiben. Bei der Todesfeier in der Kirche durften nur noch die Verwandten anwesend sein. Auf ein Todesmahl wurde verzichtet. Die Quarantäne wurde erstmals eingeführt [1, S. 187].

Erfahrungsmedizin wurde grossgeschrieben. Heilpflanzen hatten in dieser Medizin ihren festen Platz. Die Bevölkerung kannte die verschiedensten Anwendungsformen, und Rang und Stand der Menschen spielten hierbei keine Rolle. Quer durch alle Bevölkerungsschichten wurden Heilpflanzen gegen die Pest eingesetzt: innerlich wie äusserlich, über die Nahrung, als Ritual bei Alltagshandlungen sowie im Haus und im Stall zur Abwehr des Bösen. Bis zur Entdeckung des Bakteriums Yersinia pestis im Jahr 1894 und zur späteren Entwicklung des Antibiotikums waren diese Heilpflanzenanwendungen eine der wenigen aktiven Handlungsmöglichkeiten des Menschen im Kampf gegen die Pest. 
Die vorliegende Literaturstudie gibt einen Einblick in das Krankheitsverständnis und die Ausbreitungswege der Pest vom Mittelalter bis in die frühe Neuzeit. Ausserdem wird der aktuelle Kenntnisstand zur Pest gegenübergestellt. Der Schwerpunkt liegt auf der Zusammenstellung von Anwendungen von Pestpflanzen im Mittelalter.

\section{Die Pest im Mittelalter}

\section{Ausbreitung in Europa}

Die erste pandemische Ausbreitung der Pest fand bereits am Übergang zwischen Spätantike und Frühmittelalter in den Jahren 541/543 unter der Herrschaft des oströmischen Kaisers Justinian (527-565) statt (Justinianische Pest). Die zweite Pestwelle erfolgte Mitte des 14. Jahrhunderts. Es gibt verschiedene Thesen, wo die Pest ihren Anfang hatte und über welche Route sie sich nach Europa ausgebreitet hat. Eine These geht davon aus, dass die Krankheit 1330 in den zentralasiatischen Steppen ausgebrochen ist und sich entlang der Karawanenroute ausgebreitet hat [2, S. 13]. Eine andere These behauptet, dass die Pest an der Donaumündung am Asowschen Meer, einem Nebenmeer des Schwarzen Meeres, entstanden ist. Im Handelsposten Kaffa (heute Theodosia bzw. Feodossija) auf der Krim wurden genuesische Händler von den Tartaren belagert. Die Angreifer mussten sich allerdings zurückziehen, da sie an der Pest erkrankten. Hunderte Leichen blieben unbestattet zurück. Laut dem italienischen Chronisten Gabriele d' Mussis (1280-1356) liess der Tartarenführer Khan Jani Beg († 1357) die Leichen in die Katapulte laden und in die Stadt schiessen, damit die Stadtbewohner ebenfalls der Pest erliegen würden. Nicht alle Genueser starben an der Pest, sondern verschleppten sie unwissentlich über Trapezunt und Konstantinopel nach Messina und bis nach Sizilien (Herbst 1347). Von dort aus breitete sich der «Schwarze Tod» über den Land- und Meeresweg über ganz Europa aus [1, S. 221; 2, S. 13]. Schätzungsweise ein Drittel der europäischen Bevölkerung starb zwischen 1347 und 1353 an der Pest, was eine eigentliche demographische Krise mit grossen sozialwirtschaftlichen Konsequenzen war.

\section{Krankheitsverständnis}

Der Zusammenhang zwischen schlechten hygienischen Umständen, in denen die Menschen im Mittelalter lebten, und der Entstehung und Weitergabe bestimmter Krankheiten war nicht bekannt. Die Tatsache, dass Flöhe und Ratten für die Verbreitung der Pestkrankheit bei den Menschen verantwortlich sind, wurde erst im späten 19. Jahrhundert entdeckt. Das Krankheitsverständnis der
Pest im Mittelalter wurde somit von anderen Denkbildern geprägt. Da gab es die göttliche Erklärung: Die Pest wurde als Strafe Gottes für ein Leben voller Sünde verstanden. Ein Gott geweihtes Leben war die einzige Möglichkeit, um Seelenheil zu erlangen. «Tempore pestilentie penitencia et confessio medicamentis ceteris preferantur» aus dem Regimen von Kamintus [3] bedeutet, dass Reue und Beichte zur Zeit der Pest anderen Arzneien vorzuziehen sind. Ausserdem wurden auf Befehl der Kirche Freudenhäuser und öffentliche Badhäuser geschlossen. Enthaltsamkeit war angesagt, da die Sexualstoffe gemäss der Kirche zur Verunreinigung des Körpers und somit zur Entfernung von Gott führen würden [4]. Ein fundiertes Wissen in der Astrologie, als Teil des Medizinstudiums, prägte das Denken der Ärzte. Eine astrologische Erklärung für das Auftreten der Pest ging von einer ungünstigen Stellung von Planeten und Sternen aus [2, S. 14; 5]. Andere Erklärungen beriefen sich auf das Wetter oder auf Naturereignisse wie Erdbeben und Dürre.

Auch der Mensch stand teilweise im Mittelpunkt der Krankheitserklärung: Der durch den Abfall in den Strassen verursachte Gestank, die Misthaufen, die Ausdünstungen, der Atem, die Kleider und die Beulen der betroffenen Menschen kamen als mögliche Ursache für die Seuche ins Gerede [2, S. 4].

\section{Diagnostik, Behandlung und Prophylaxe}

Im Mittelalter war die Diagnostik der Pestkrankheit nur anhand der klinischen Erscheinungsformen möglich. Hohes Fieber, kombiniert mit dem Auftreten der Beulen, gab die unheilsame Gewissheit um die Anwesenheit der Krankheit in einer Stadt, in einer Familie oder bei der einzelnen Person. Die Triage "Ansteckung-Inkubationszeit-klinische Erscheinung» war aufgrund der damaligen

Abb. 1. «Doktor Schnabel von Rom». Stich, Rom, 1656. Schutzkleidung von Ärzten gegen die Beulenpest bzw. den «Schwarzen Tod» (Quelle: Commons. Wikimedia.org).

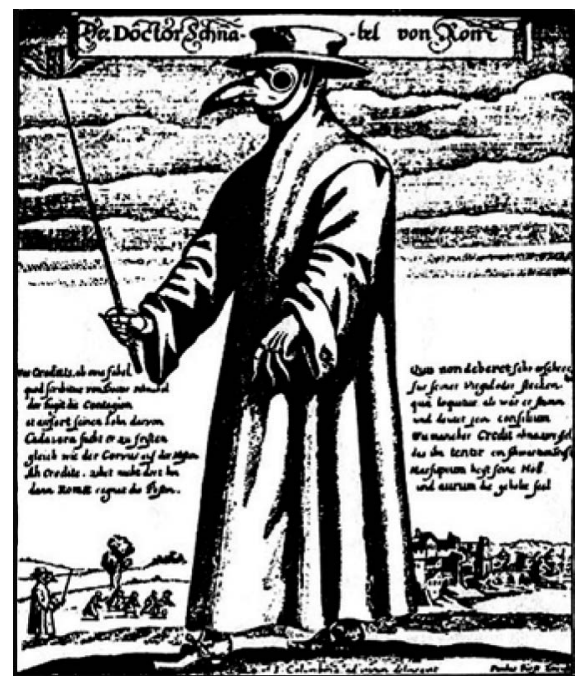

Wulfers 


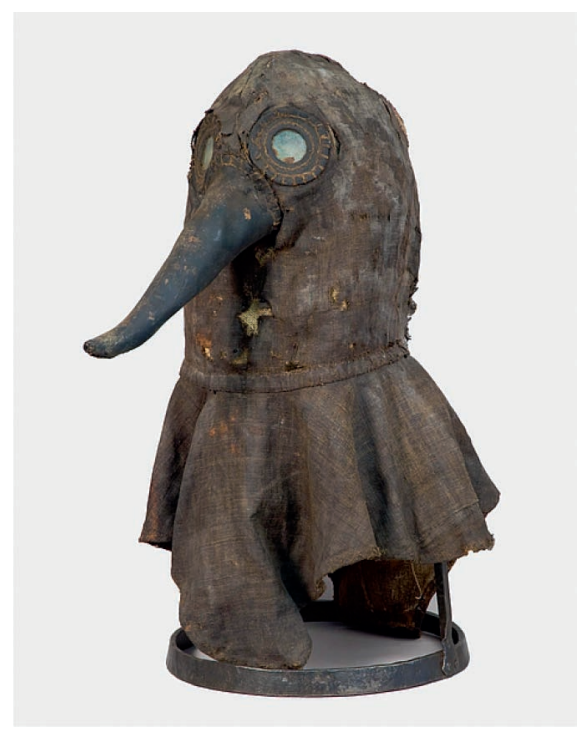

Abb. 2. Pestarztmaske (Stoff, Leder, Glas; um 1700; Masse $61 \times 36 \times 45 \mathrm{~cm}$ ). Abdruck mit freund licher Genehmigung des Deutschen Medizinhistorischen Museums Ingolstadt, Deutschland (Foto: Michael Kowalski).

medizinischen Kenntnisse kein Begriff. Aus der Empirik heraus entstanden jedoch «Vorsorgemassnahmen», um die Verbreitung der Krankheit einzudämmen. Doktoren hüllten sich in dicke Kleider ein (Abb. 1) und trugen eine Pestmaske vor dem Gesicht (Abb. 2). In der Pestmaske war ein mit Essig getränktes Tüchlein mit pulverisierten Kräutern, um die Ansteckung mit der Pestkrankheit $\mathrm{zu}$ verhindern. Teilweise kaute man die Wurzeln der Engelwurz (Angelica archangelica L.) (Abb. 3) [1, S. 41]. Krankenzimmer wurden ausgeräuchert, um die «faulige» Luft zu vertreiben, und die Kleider der Pestkranken wurden im Dampf eines Kräutersuds gereinigt. Die Häuser der Pesttoten wurden ausgeräuchert oder mit Kräuteressig desinfiziert. Post wurde geräuchert und das Geld desinfiziert [4]. Die Leichenwäscher gebrauchten Gamander (Teucrium scordium L.), der als starkes Mittel gegen die Pest galt $[1$, S. 123].

Die grossen Städte schlossen ihre Eingangstore und Häfen. Die Reisenden, Handels- und Schiffsleute mussten 40 Tage vor den Pforten der Stadt bzw. vor dem Hafeneingang ausharren. Wäre jemand mit der Pest infiziert, so würde er diese 40 Tage nicht überleben. So entstand das Prinzip der Quarantäne (ital. quaranta giorni), das wir auch heutzutage noch kennen [2, S. 18].

\section{Pest in der heutigen Zeit}

\section{Krankheitserreger}

Das Bakterium Y. pestis wurde im Jahr 1894 vom Schweizer Bakteriologen Alexandre Émile Jean Yersin ( 22.9.1863 Aubonne/Schweiz - † 28.2.1943 Nha Trang/ Vietnam) entdeckt. Ursprünglich wurde das Bakterium nach Louis Pasteur, dem Lehrmeister von Yersin in

Paris, benannt (Pasteurella pestis). Zu Ehren seines Entdeckers wurde es später umbenannt [6]. Pestis bedeutet auf lateinisch «die Seuche», «die ansteckende Krankheit».

Das Bakterium Y. pestis gehört zu der Familie der Enterobacteriaceae. Es ist gramnegativ, stäbchenförmig und je nach «Bedarf» aerob oder intrazellulär [7]. Die Körpertemperatur des Flohs, einem Überträger des Pestbakteriums, beträgt $24^{\circ} \mathrm{C}$. Bei dieser Temperatur würde das Bakterium von Leukozyten und Monozyten vernichtet werden. Bei Körpertemperaturen von $37^{\circ} \mathrm{C}$ wie beim Menschen oder von $38,5^{\circ} \mathrm{C}$ wie bei den Ratten bildet das Bakterium jedoch eine Kapsel von Proteinen um sich herum. Diese schützt das Bakterium gegen Angriffe des Immunsystems (Phagozytose). Zusätzlich hat es die Fähigkeit, intrazellulär einzudringen, um sich dort, aufgrund der nährstoffreichen Umgebung, zu vermehren [7]. Dieser Schutzmechanismus ist $3 \mathrm{~h}$ nach Eintritt vorhanden. Der Unterschied in der Körpertemperatur zwischen Floh und Ratte steigert die Virulenz des Bakteriums um das 50-Fache. Die Umgebungstemperatur und der Untergrund sind dafür entscheidend, wie lange das Pestbakterium überlebensfähig ist. Bei Raumtemperatur und normaler Luftfeuchtigkeit ist das Bakterium während mehr als 5 Tagen ansteckend [8]. Das Bakterium wird durch Erhitzen - bei Temperaturen über $55^{\circ} \mathrm{C}$ während 15 min - inaktiviert [9].

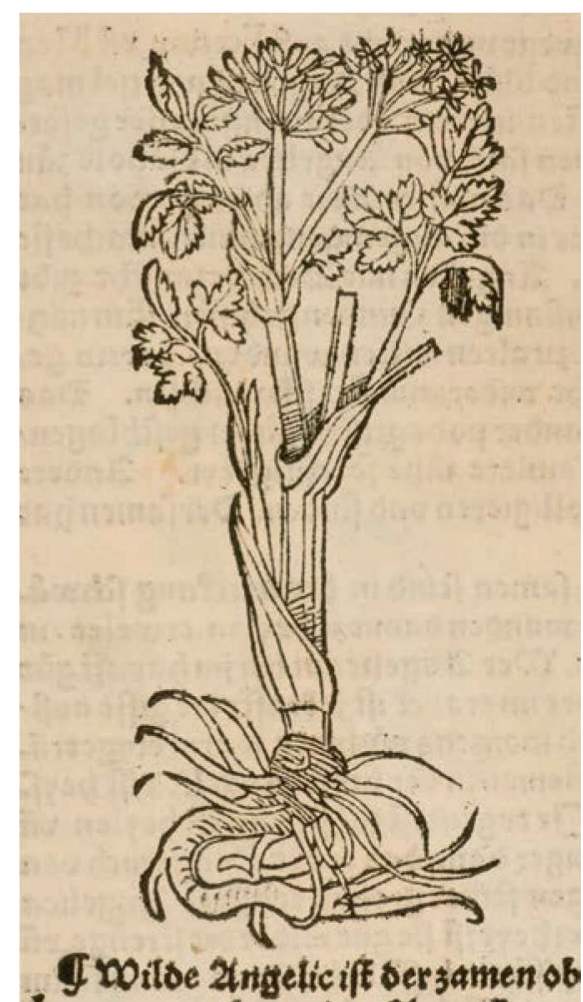




\section{Übertragungsweg}

Bei der Übertragung der Pestkrankheit spielen Flöhe, speziell der Rattenfloh (Xenopsylla cheopsis), eine bedeutende Rolle. Flöhe sind Parasiten und benötigen einen Wirt zum Überleben. Sie nehmen beim Blutsaugen an einem infizierten Tier Bakterien auf und können ihren nächsten Wirt erneut mit diesen Bakterien infizieren. Die Weitergabe des Pestbakteriums verläuft auf diesem Weg, indem der Floh als «Zwischenwirt» für das Pestbakterium fungiert. Ratten, aber auch andere wilde und häusliche Nagetiere (Eichhörnchen, Erdhörnchen, Hasen, Hunde, Katzen) können Träger des Pestbakteriums sein. Sterben zu viele Ratten an der Pest, so weichen die Flöhe auf andere Gastwirte aus und infizieren diese. Etwa 30 Floharten, inklusive des Menschenflohs (Pulex irritans L.), eignen sich als Überträger des Pestbakteriums. Der Mensch wird zum «Fehlwirt» und somit mit dem Bakterium $Y$. pestis infiziert. Hat die Seuche den Mensch erreicht, findet die Ansteckung direkt von Mensch zu Mensch statt. Dies erfolgt über die verwundete Haut, über Schleimhautverletzungen im Mund und Rachenraum sowie über Sekret oder Fäkalien. Bei der Lungenpest findet die Ansteckung aerosol mittels Tröpfcheninfektion statt. Die Kontaminierung des Trinkwassers, der Lebensmittel oder medizinischer Materialien, wie einer Injektionsnadel, können ebenfalls zu einer Ansteckung führen. Das Pestbakterium überlebt jedoch auch ohne den menschlichen oder tierischen Gastwirt. Es schlummert längere Zeit in Kot, in der Erde, in Kadavern oder in Kleidern und ist jederzeit in der Lage, neue Opfer zu infizieren [5, S. $4 ; 9]$.

\section{Pest - Krankheitsformen und Symptome}

Die Symptome der Pestkrankheit sind je nach Erscheinungsform unterschiedlich. Die drei am häufigsten vorkommenden Krankheitsformen sind:

\section{Beulen- oder Bubonenpest}

Die Beulenpest (lat. bubo $=$ Beule) ist die häufigste Erscheinungsform der Pestkrankheit. Durch die Einstichstelle des mit dem Pestbakterium infizierten Flohs dringen Bakterien in den Körper ein, die die Lymphknoten befallen. An der Bissstelle kann es zu Pusteln, Blasen oder schorfigen Stellen kommen. Meistens sind primär die Lymphknoten der Leistengegend (65-75\%) betroffen. Dies hat mit der eingeschränkten Sprungweite des Flohs zu tun, der maximal $30 \mathrm{~cm}$ hoch und $50 \mathrm{~cm}$ weit springen kann. In 10-20\% der Fälle tritt die Primärbeule in den axillären Lymphknoten auf. Selten ist der Primärbefall in der Halsgegend (5-10\%) zu finden. Die Inkubationszeit beträgt 1-7 Tage. Das erste allgemeine Symptom der Beulenpest ist hohes Fieber bis zu $41{ }^{\circ} \mathrm{C}$, das kaum mehr zu senken ist. Der Patient ist schwer krank: Kopf- und Gliederschmerzen, Schüttelfrost, Schwindelgefühl, reduzierte Ansprechmöglichkeit und starkes Durstgefühl trotz verminderter oder ausgesetzter Harnausscheidung. Die Beulen erscheinen erst 24 h nach Krankheitsbeginn. Zuerst sind sie hart und sehr druckempfindlich. Nach einiger Zeit füllen sie sich aufgrund der körpereigenen Abwehrstoffe mit Eiter und werden etwas weicher. Die Haut über der Beule ist gerötet, gespannt und erwärmt. Rundherum entsteht ein schwarz-bläuliches Ödem durch die inneren Blutungen der Lymphknoten. Die Beulen haben einen Durchmesser von 1-10 cm. Unbehandelt führt die Beulenpest innerhalb von 1-7 Tagen zum Befall anderer Organe und damit zum Tod [9].

\section{Lungenpest}

Die Lungenpest tritt primär auf, wenn Menschen bakterienhaltige Tröpfchen einatmen. Der Abstand zum Gesicht einer infizierten Person ist entscheidend, ob eine Ansteckungsgefahr besteht. Das Pestbakterium stirbt an der Luft rasch ab. Befindet man sich nahe am Gesicht eines Patienten (innerhalb von $30 \mathrm{~cm}$ ), so ist die Ansteckungsgefahr gross. Die Symptome einer Lungenpest sind atypisch und können zuerst mit einer Lungenentzündung verwechselt werden - vor allem, weil bei der primären Lungenpest keine Beulen auftreten. Die Krankheit beginnt mit allgemeinen Fiebersymptomen wie Schüttelfrost, Abgeschlagenheit und Schwindel. Husten, Atemnot, Brustschmerzen und Zyanose treten am zweiten Tag auf. Es entsteht ein schwarzblutiges, hochinfektiöses Sekret. Das Aufhusten ist extrem schmerzhaft. Die Krankheit kann zusätzlich von gastrointestinalen Symptomen wie Durchfall, Erbrechen, Bauchschmerzen und Übelkeit begleitet werden. Das Lungenödem führt rasch zu einem Herz-Kreislauf-Versagen und damit zum Tod. Die Inkubationszeit beträgt einige Stunden bis zu 4 Tagen. Da die Lungenpest atypisch verläuft und die Diagnose häufig verpasst wird, ist die Sterblichkeitsrate bis zu 95\% hoch. Eine sekundäre Lungenpest tritt als Komplikation einer unbehandelten Beulenpest auf $[4,10]$.

\section{Pestsepsis}

Wenn der Pesterreger in die Blutbahn gelangt, entsteht eine Blutvergiftung (Pestsepsis). Das Bakterium verteilt sich in alle Organe, was zu hohem Fieber mit Lethargie, Bewusstseins- und Orientierungsstörungen, Darmverschluss und septischem Schock führt. Im Endstadium 
werden Zellen in den distalen Körperteilen aufgrund der Thrombose in den kleinen Endarterien schlecht durchblutet. Dies führt zur Zellsterblichkeit in Zehen-, Fingerund Nasenspitze. Die Pestsepsis tritt primär auf, wenn das Blut direkt über offene Wunden infiziert wird. Der Patient stirbt meistens, bevor die Krankheitssymptome sich manifestieren. Eine sekundäre Blutvergiftung tritt als Komplikation einer Beulen- oder Lungenpest auf [4].

Die Pestmeningitis (Hirnhautentzündung) und die Pestpharyngitis (Rachenentzündung) sind selten und treten als Komplikationen anderer Pestformen auf. Die abortive Pest ist eine milde Form der Pest mit geringer Schwellung der Lymphknoten und leichtem Fieber. Unbehandelt verschwinden die Symptome nach einer Woche. Der Patientbildet Antikörper, was einelangfristige Immunität gegen die Krankheit gewährleistet.

\section{Aktuelle Verbreitung der Pest}

Seit Alexandre Yersin 1894 das Bakterium Y. pestis entdeckt hat, sind die Entstehung der Pestkrankheit und die Übertragungswege geklärt. In Mitteleuropa besteht fast keine Pestgefahr mehr. Dies bedeutet jedoch nicht, dass das Bakterium ausgerottet ist. Auf folgenden Kontinenten kommt die Pestkrankheit noch vor:

- Afrika: südlich der Sahara.

- Asien: in den ehemaligen Sowjetstaaten Zentralasiens, in Indien, in China, in der Mongolei sowie in Myanmar, Laos, Vietnam und Indonesien.

- Süd- und Mittelamerika: in Ecuador, in Peru, in den Anden und im nordöstlichen Brasilien.

- USA: Im Südwesten treten nur noch sporadisch Fälle der Pestkrankheit auf [9].

\section{Pestdiagnostik, -behandlung und -prophylaxe}

Wenn keine Beulen entstehen, geben auch heutzutage die Symptome nicht sofort den Hinweis auf die Pestkrankheit. Zur Differentialdiagnose gehören auch Malaria, Q-Fieber, Fleckfieber, Brucellose und Typhus. Wenn Beulen auftreten, wird der Patient zusätzlich auch auf Diphterie und Tularämie untersucht. Spezielle Diagnoseverfahren bestätigen das Vorhandensein von Y. pestis. Hierfür wird Blut, Sputum oder Eiter aus den Lymphknoten benötigt. Bei $28{ }^{\circ} \mathrm{C}$ werden Bakterienkulturen gezüchtet. Y. pestis wächst nur langsam, sodass erst nach $48 \mathrm{~h}$ das Resultat bekannt wird. Unter dem Mikroskop sieht man ein kurzes, eiförmiges Bakterium. Färbt man die Bakterien mit Methylenblau ein, so wird eine Sicherheitsnadelstruktur sichtbar, da die Vakuole im Bakterium nicht eingefärbt wird. Diese Bipolarität bestätigt den Verdacht auf die Infizierung mit $Y$. pestis. Der Nachweis von Antigenen im Blut ist auch aussagekräftig, ob eine Infizierung stattgefunden hat oder nicht [4].

Bei Verdacht auf eine Pesterkrankung wird sofort mit einer Antibiotikabehandlung (Streptomycin, Gentamycin) begonnen, bevor die Laborresultate vorhanden sind. Die Erkrankung an der Pest ist in der Schweiz meldepflichtig beim Bundesamt für Gesundheit (BAG). Die Lungenpest ist hochinfektiös; erkrankte Personen müssen daher sofort isoliert werden. Die Behandlung muss innerhalb von $24 \mathrm{~h}$ nach Erscheinen der ersten Symptome begonnen werden. Bei rechtzeitiger Behandlung mit Antibiotika sterben nur noch $10 \%$ der Patienten. Eine zu spät erfolgte Behandlung oder deren Unterlassung führen zu 100\% zum Tod. Bei der Beulenpest ist eine Übertragung heutzutage nur noch möglich, wenn die Beulen aufplatzen und infektiöser Eiter aus der Wunde tritt. Die Heilungschancen sind deutlich besser als bei der Lungenpest. Unbehandelt sterben $60 \%$ der Patienten, während diese Zahl mit Behandlung auf 5\% reduziert wird. Eine unbehandelte Pestsepsis führt auch heutzutage unweigerlich zum Tod. Bei rechtzeitiger Behandlung reduziert sich die Todesrate auf 33\% [9].

Reist man in Gebiete mit aktivem Pesterreger, so ist auf saubere, hygienische Lebensumstände $\mathrm{zu}$ achten. Kontakte mit Flöhen, Ratten und streunenden (Haus-) Tieren sollten vermieden werden. Ebenso sollten mitreisende Haustiere durch ein Antiflohmittel geschützt werden. Es gibt zwei Totimpfstoffe (Haffkine-Vakzine und Cutler-Vakzine), wobei der Schutz nur gegen die Beulenpest gilt. In der Schweiz ist die Impfung nicht zugelassen. Obwohl in den betroffenen Gebieten teilweise Impfstoffe vorhanden sind, rät das BAG von dieser prophylaktischen Impfung ab, da die Wirksamkeit umstritten ist [9]. Seit dem 10. Januar 2006 gibt es einen neuen deutsch-amerikanischen Impfstoff, der gute Resultate zeigt. Aus gentechnisch veränderten Tabakpflanzen wurden immunogene Proteine für den Impfstoff gewonnen [4].

\section{Anwendung von Heilpflanzen als Mittel gegen die Pest}

\section{Applikationsformen}

Im Mittelalter gab es keine eindeutigen Kenntnisse über die Krankheitsentstehung. Die Symptome und die Folgen der Pest wurden den Menschen aber ungewollt sehr schnell vor Augen geführt. Die Pest wütete in Europa, sodass den Menschen nichts anderes übrig blieb, als ihr «den Kampf anzusagen». Heilpflanzen spielten in der medizinischen Behandlung der Pest eine sehr grosse Rolle. Die Applikationsform der Heilpflanzen wurde durch die klinischen Symptome bestimmt. Umschläge 
gab es auf Beulen - zum Aufweichen oder wenn sie anfingen zu eitern. Einreibungen machte man mit Essig, der mit verschiedenen Heilpflanzen angereichert war. Es wurde gedämpft, geräuchert, gesalbt, getrunken und gegessen, um der Pest Herr zu werden. Musik, auf einer Zither gespielt, die aus dem Holz des Weinstocks oder des Lorbeers fabriziert wurde, sollte die Ansteckung durch die Pest verhindern: "Contra pestilentiam cithara usus est, quod non nisi ex vitigineo ligno esse poterat, quum mire, und acetum contra pestilentiam valeant. Vel ex lauro, cuius folia tusa, und olfacta, subinde pesti lentia contagia prohibeant» [11].

Es wurden Blüten, Blätter, Beeren, Wurzeln, Samen und Harz gebraucht. Die Heilpflanzen wurden in verschiedensten Formen angewendet: frisch, getrocknet, pulverisiert oder eingelegt in Essig, Alkohol, Wein oder Öl. Die Medizinalgetränke wurden sowohl kalt als auch warm getrunken.

Heutzutage interessieren uns vor allem die Inhaltsstoffe einer Pflanze, sind diese doch entscheidend für das Wirkungsfeld. Im Mittelalter waren die chemischen Inhaltsstoffe einer Pflanze jedoch noch nicht erforscht, sodass die Wirkungsart der Pflanze im Mittelpunkt stand. Ob eine Pflanze schmerzlindernd, schweisstreibend, durststillend, harnausscheidend oder herzstärkend war, bestimmte den Einsatz gegen die Pest. Es gab Mono-Anwendungen oder Kombinationsanwendungen verschiedener Heilpflanzen. Ansätze, die wir aus der Homöopathie kennen, wurden auch dazumal, bewusst oder unbewusst, schon zelebriert: «Stinkende

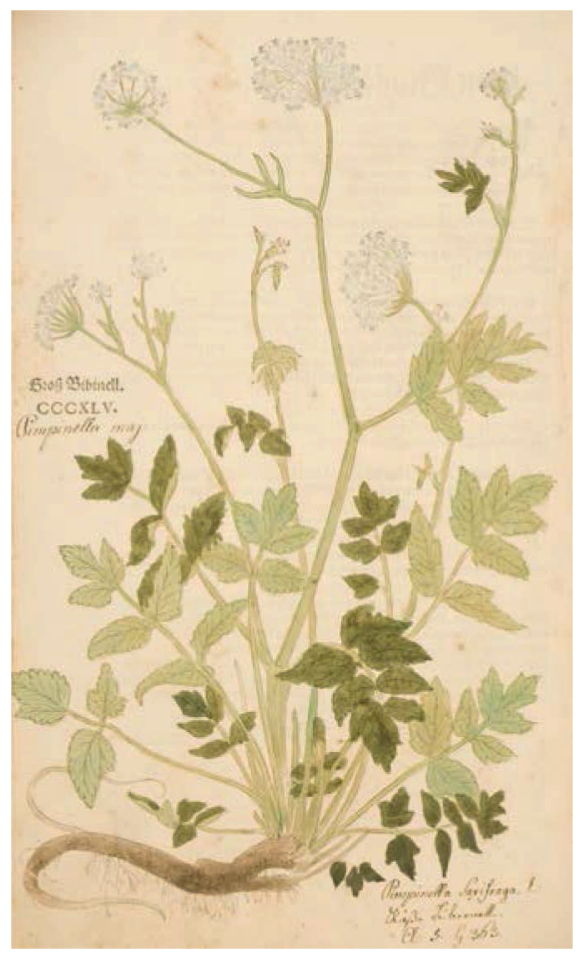

Abb. 4. Bibernelle (Pimpinella saxifraga) (New Kreüterbuch, 1543).

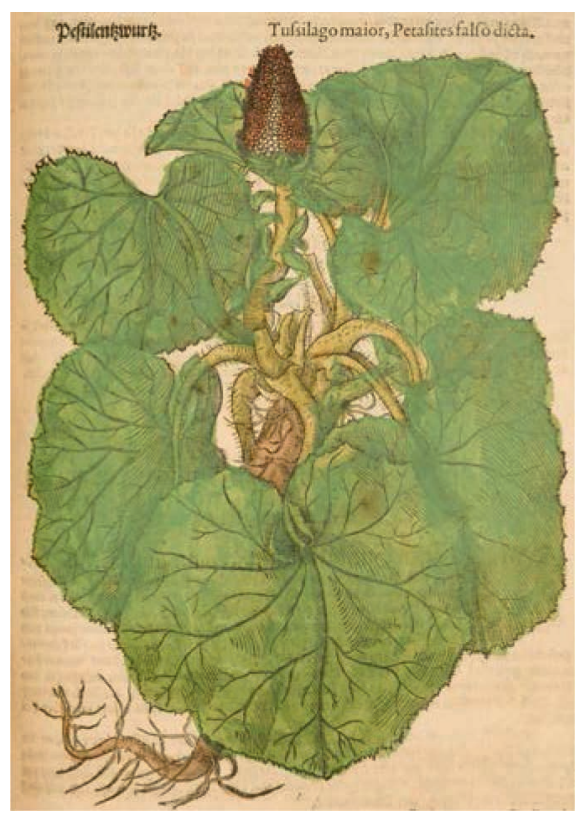

Wurzeln vertreiben die stinkende Pest» (Gleiches mit Gleichem vertreiben). Dies galt auch für die Wurzel des Baldrians (Valeriana officinalis L.).

Die Auswahl an Heilpflanzen war gross. In zahlreichen Sagen und Legenden dieser Zeit kamen Engel, Vögel oder andere Waldwesen vor, die dem Menschen verraten sollten, welche Pflanzen gegen die Pest gebraucht werden können. Am häufigsten werden die Bibernelle (Pimpinella saxifraga L.) (Abb. 4) und der Wacholder (Juniperus communis L.) genannt [1, S. 45]. «In einem Brief von Konrad Gessner an Kosmas Holtzsch vom 24. September 1564, während dem die Pest in Zürich wütete, fragte er diesen: «Hast du zufällig jenen Vers gehört, der vom Himmel - man höre! - stammen soll: nemmend Bibernell, und so gnaesend ir schnell, worunter nur Bocksteinbrech zu verstehen ist.»" [1, S. 55].

\section{Äussere Anwendungen}

\section{Umschläge}

Von Hieronymus Bock gibt es ein Rezept mit Tollkirsche (Atropa belladonna L.), womit er gerade zwei Symptome der Pest beschreibt: «Kraut, Blüten und reife Früchte als Destillat gegen «hitzige Presten〉 (fieberhafte Krankheiten, auch Pest) ... grüne Blätter als Umschlag oder Destillat als Einreibung gegen «Gross hitzig hauptwehe> (Kopfschmerzen bei der Pest)» [1, S. 117].

Schweisstreibende Umschläge wurden gemacht zum «kühlen bei Menschen im delirierenden Pestfieber» und gegen «das wueten der schelligen Menschen» $[1$, S. 127]. Folgende Pflanzen wurden dafür verwendet: Winterendivie (Cichorium endivia L.), Fluss-Ampfer (Rumex hydro- 
lapathum Camd.) und Saat-Mohn (Papaver dubium L.) [1, S. 118, 122, 127].

Das «grausam hauptwee» (Kopfweh) war ein Zeichen des Krankheitsbeginns. Umschläge gegen Kopfschmerzen wurden gemacht mit Tollkirsche (A. belladonna L.), Spitzwegerich (Plantago lanceolata L.), Flaschenkürbis (Lagenaris vulgaris Ser.) und Wald-Ehrenpreis (Veronica officinalis L.) [1, S. 37, 108, 117, 131].

Die Pestbeulen, die 24 h nach Krankheitsbeginn entstehen, sind zuerst sehr hart und schmerzhaft. Zur Schmerzlinderung und Aufweichung der Beulen wurden Umschläge mit folgenden Heilpflanzen empfohlen: Knollen-Hahnenfuss (Ranunculus bulbosus L.), Weisse Mistel (Viscum album L.), Grüne Nieswurz (Helleborus viridis L.), Gewöhnliche Natternzunge (Ophioglossum vulgatum L.), Benediktenkraut (Cnicus benedictus L.), Einbeere (Paris quadrifolia L.) und Echte Pestwurz (Petasites hybridus L.) (Abb. 5) [1, S. 19, 25, 45, 105, 106, 135].

\section{Einreibungen}

Einreibungen mit Essig wurden gemacht, um die Menschen vor der Ansteckung mit der Pest zu schützen. Der Pestessig der vier Diebe von Marseille und Toulouse ist wohl eines der berühmtesten Einreibmittel [12]. Ein Bestandteil davon war unter anderem Nelkenwurz (Geum urbanum L.). Öl aus den Samen des Bilsenkrauts (Hyoscyamus albus bzw. niger L.) wurde unter anderem durch Albertus Magnus «für grosse Hitz im Haupt» empfohlen. Gamander (T. scordium L.) wurde von den Leichenwäschern zum Schutz gegen die Pest gebraucht $[1$, S. 29, 92, 123].

\section{Badezusatz}

Das Baden im Absud der Osterluzei (Aristolochia rotunda L.) wurde bei chronischen Geschwüren und Eiterungen empfohlen [1, S. 127].

\section{Salbe}

Salben wurden benötigt, um die Beulen aufzuweichen. Platzten diese auf, so wurden die Salben gegen die Eiterungen und zur Wundheilung aufgetragen. Rochus, der Schutzpatron gegen die Pest, wird auf einem um 1480 entstandenen Holzschnitt mit einer Pestbeule am Oberschenkel dargestellt und mittels einer Salbe behandelt. Hildegard von Bingen empfiehlt ein «Feder von Tollkirschsaft (Atropa belladonna L.)» in Hirsch- und Gänseschmalz aufgelöst als Salbe bei «grossen und sehr scharfen d.h. durchsoden Geschwüren» [1, S. 117]. SumpfHaarstrang (Peucedanum palustre (L.) Moench) wurde 1587 von Leonhardt Thurneyssen als Teil eines Balsams empfohlen, der auf Zunge, Nase, Ohren und Schlafadern aufgetragen wurde gegen die "pestilentzische Kranckheiten» $[1$, S. 130].

\section{Pflaster}

Die Pflaster wurden auf die Pestbeulen gelegt mit dem Ziel, diese aufzuweichen. Waren die Pestbeulen nicht mehr so hart, brachen sie vielfach auf. Damit wurden die Schmerzen etwas erträglicher. Schöllkraut (Chelidonium majus L.) wurde ausführlich von Tabernaemontanus als heilende Substanz in einem Pflaster aus «Saltz und Schweineschmaltz» beschrieben [1, S. 129]. Beinwell (Symphytum officinalis L.), Gundelreben (Glechoma hederacea L.) und Weihrauch (Boswellia carterii Birdw.) wurden ebenfalls als Pflaster-Zutaten empfohlen [1, S. 43, 84, 98].

\section{Amulette}

Das Amulett diente zur Abwehr der Pestkrankheit und schützte die Person, die das Amulett trug. Die andere Möglichkeit war, dass das Amulett die Krankheit an sich zog, sodass die Person, die das Amulett trug, nicht erkrankte. Eine Reisebeschreibung aus dem Elsass erwähnt: "Viele Leute haben mir versichern wollen, dass die Zwiebel gar ein Präservative gegen die Pest sey, wenn man sie an sich trüge oder häufig in seinem Hause aufhänge, sie zöge die vergiftete Luft an sich, und würde bald Schwarz davon» [1, S. 71]. Amulette gab es in verschiedenen Formen und Ausführungen. Es gab das Amulett als Schmuck (z.B. eine Kette aus Nelken oder gepressten Blüten der Deutschen Schwertlilie) oder in «Rohform» als Wurzel. Diese Wurzel konnte angenehm duften wie die Veilchenwurzel (Iris florentina L.) oder «stinken wie die Pest» wie die Baldrianwurzel.

Wurzeln folgender Heilpflanzen wurden als Amulett getragen: Echte Betonie (Stachys officinalis Franch.), Gefleckter Aronstab (Arum maculatum L.) und Bibernelle. Tabernaemontanus schreibt in seinem «New Kreuterbuch»: «die gemeldte Wurzel auf blosser Haut am Halss getragen/soll den Menschen bewahren/dass ihn die Pestilenz nicht anstosse» [1, S. 58]. Hiermit ist die Bibernelle gemeint.

\section{Riechapfel}

In einem Bisamapfel oder Riechapfel befand sich ein Stücklein Stoff oder ein Schwämmlein, das in einem Sud von pestabwehrenden Heilpflanzen getränkt wurde [13]. Dies sollte die Person, die den «Bisemapffel» trug, gegen die Pest schützen. Aloe (Aloe vera L.), Weihrauch (B. carterii Birdw.), Kalmus (Acorus calamus L.) und das Harz des Pistazienbaums (Pistacia lenticus L.) waren nur einige von vielen Heilpflanzen, die, meistens in Kombination, für den Riechapfel gebraucht wurden [1, S. 98].

\section{Räucherrituale}

Im Pestbuch von Heinrich Steinhöwel aus dem Jahr 1473 wurde Folgendes empfohlen: «füwer machen aus 
wecholdern, rossmarin, aychen usw. denn diese ding reinigen den lufft von syner fülin (Fäulnis) durch ir werme» $[1$, S. 80].

Das Räuchern hatte eine reinigende Bedeutung. In den Städten wurde mittels grosser Feuer geräuchert, z.B. mit dem Holz des Wacholders [14]. In den Häusern und Pesthäusern (Krankenhäuser speziell für Pestkranke) fand das Räuchern in kleinem Ausmass statt. Das Ausräuchern der Kleider wurde von den einzelnen Personen vorgenommen. Es wurde sowohl mit Harzen als auch mit getrockneten, pulverisierten Teilen (Blätter, Wurzel, Rinde) bestimmter Heilpflanzen geräuchert. Folgende Pflanzen wurden zum Räuchern gebraucht: Myrrhe (Harz von Commiphora schimperi Engl.), Weihrauch (Harz von B. carterii Birdw.), Gemeiner Wacholder (Beeren und Holz von J. communis L.), Bibernelle (Wurzel von P. saxifraga L.) und Meisterwurz (Wurzel von Peucedanum ostruthium W.D.J. Koch).

\section{Kräuterbüschel}

Die Kräuterbüschel bestanden aus geruchsstarken Heilpflanzen wie Knoblauch (Allium sativum L.), Gemeiner Schafgarbe (Achillea millefolium L.), Bibernelle (P. saxifraga L.) sowie Gemeiner Wacholder (J. communis L.) und dienten dazu, "Haus und Hof» gegen die Pest zu schützen. Sie dienten als Filter gegen das Böse [1, S. $21,46,71,77]$.

\section{Innerliche Anwendungen}

\section{Gebranntes «Wasser»}

Die Pestkrankheit verursacht Fieber. Dies lässt den Patienten stark schwitzen, was zu einem hohen Flüssigkeits- und Elektrolytverlust führt. In verschiedenen Rezepturen wird auf durststillendes «Wasser» hingewiesen. Dabei ist mit Wasser nicht das Trinkwasser, wie wir es heutzutage kennen, gemeint, sondern die meisten Rezepturen erwähnen ein Wasserdestillat angereichert mit Wein oder sonstigem Alkohol, besser bekannt als Theriak. Theriak wurde nicht nur als prophylaktisches Mittel gegen die Pest getrunken, sondern auch bei starkem Durstgefühl oder als Schwitzkur, um den Körper von den Giften zu befreien. Heilpflanzen wie Gemeiner Erdrauch (Fumaria officinalis L.) und Buchampfer (Oxalis acetosella L.) waren ein Bestandteil des durststillenden Getränks $[1$, S. 35, 119].

Tabernaemontanus schreibt über das Schöllkraut als Schwitzkur Folgendes: «Schellkrautwurtzel gesäubert und klein geschnitten/einer Hand voll in einer halben Mass Rosenessig auf das halbe Theil eingesotten/darnach druchgesigen/und in der Brühe zertrieben 3. Loth guten Alexandrinischen Theriack/und dieses Tranks ein Pfennig-Glässlein voll getrunken/niederlegen/und biss in die
3. Stunden geschwitzet/den Schweiss darnach mit warmen Tüchern abgetrucknet/ist eine bewährte Arzney/so jemand die Pestilentz hätte angestossen» [1, S. 129].

Verschiedenste Heilpflanzen wurden für den Theriak verwendet, einzeln oder in Kombination miteinander, um so die schweisstreibende Wirkung noch $\mathrm{zu}$ verstärken: Grosse Klette (Arctium lappa L.), WaldEhrenpreis (V. officinalis L.), Engelwurz (A. archangelica L.), Bibernelle (P. saxifraga L.), Weinraute (Ruta graveolens L.), Gemeiner Wacholder (J. communis L.) und Baldrian (V. officinalis L.) [1, S. 37, 40, 58, 65, 81, 136, $112]$.

\section{Salz}

Salz wurde mit Substanzen aus Heilpflanzen angereichert. Ob dies getrocknete und zerkleinerte Pflanzenteile oder Tropfen von Frischpflanzensaft waren, lässt sich anhand der Texte nicht eruieren. Beschrieben werden Wermuthsalz (Sal Absinthii) und Erdrauchsalz (Sal Fumariae) [1, S. 119, 133].

Über die Angelikawurzel (A. archangelica L.) wird Folgendes geschrieben: «Das Saltz so man aus der Angelickwurzel aussziehet/ist ein heilsam Artzeney wider die Pestilentz» [1, S. 42].

$\ddot{O} l$

Öl war als «Trägerstoff» für Heilpflanzen geeignet; daraus wurde dann ein Auszug gemacht. Von der Weissen Lilie (Lilium candidum L.) wurden die Blütenblätter gebraucht. So entstand «der weisse Lilien-Oehl». Aus dem Holz des Gemeinen Wacholders (J. communis L.) wurde das Wacholderöl hergestellt. Kampheröl war eine Kombination aus Agstein- und Citronrindenöl kombiniert mit Kampfer (Cinnamomum camphora L.). Das Terpentinöl wurde direkt von einem kleinen Baum namens Terpentin-Pistazie (Pistacia terebinthus L.) gewonnen. Das Öl wurde tropfenweise eingenommen, manchmal sogar erhitzt $[1$, S. 81, 110, 121].

\section{Pillen}

Pillen wurden als «Praeservatif vor die Pestilenz» oder zur Behandlung der Symptome hergestellt und eingenommen. Hildegard von Bingen beschreibt eine Rezeptur mit Echter Aloe (A. vera L.), Myrrhe (Commiphora abyssinica Engl.) und Safran (Crocus sativus L.). Tabernaemontanus beschreibt Wermut (Artemisia absinthium L.) in Kombination mit anderen Heilpflanzen wie Salbei (Salvia officinalis L.) und Rauten (R. graveolens L.). Nostradamus wurde bekannt mit seinen «Rosenpillen». Diese Pillen bestanden aus 300-400 vor dem Morgengrauen gepflückten Rosenblättern in Kombination mit anderen Heilpflanzen sowie mit Sägespänen der Zypresse. Die Rosenpillen sollten den schlechten Atem töten und Fäulnis im Mundraum entgegenwirken. Nostradamus 
war überzeugt, dass die Pest über schlechte Luft verbreitet wurde $[1$, S. 110].

\section{Pulver}

Pulver wurde aus verschiedenen getrockneten Pflanzenteilen hergestellt. Zusammen mit Essig, Theriak oder Wein wurde es in kleinen Mengen eingenommen. Pulver wurde unter anderem aus getrockneten Lorbeeren ( $\mathrm{Lau}$ rus latifolia Salisb.), der getrockneten Wurzel des Gelben Enzians (Gentiana lutea L.) oder der getrockneten Wurzel des Blutwurz (Potentilla erecta Hampe.) gemacht $[1$, S. 30, $68,89]$.

\section{Frischpflanzensaft}

Der Saft aus verschiedenen Pflanzenteilen (Kraut, Wurzel, Samen) wurde direkt verwendet oder in Alkohol aufgelöst. Rezepturen mit Gewürznelken (Syzygium aromaticum Merr. und L.M. Perry), Königskerze (Verbascum thapsus L.), Blutwurz (P. erecta Hampe.) und Aronstab (A. maculatum L.) werden beschrieben $[1$, S. 58, 69, 91, 131].

\section{Kauware}

Wurzeln, Beeren und Holz bestimmter Heilpflanzen wurden als Kauware gegen den schlechten, krank machenden Atem eingesetzt. Matthiolus schreibt über Myrre (C. abyssinica Engl.): «Myrrhe wirt nützlich gekewet wider den stinckenden Athem Item zu sterbensluefft im Mund gehalten» [1, S. 100]. Ebenso empfiehlt er die Wurzel der Bibernelle (P. saxifraga L.) als Kauware. Die Beeren des Gemeinen Wacholders (J. communis L.) wurden von Pierre Magnolius aus Montpellier als Mittel gegen die Pest empfohlen [1, S. 81].

\section{Nahrungsmittel}

Mit Teilen von Heilpflanzen angereicherte Nahrungsmittel sollten den Menschen vor Ansteckung schützen. Tabernaemontanus schreibt: «Grüne oder frische Eisenkrautblätter des Morgens nüchtern vor sich selbst/oder auf ein Bissen Brots und gesaltzenen Butter geessen/ist ein herrlich Praeservatif vor der Pestilentz zu verhüten» [1, S. 39].

Pulver der getrockneten Angelikawurzel (A. archangelica L.) wurde ebenfalls mit Brot eingenommen. Die frischen Blätter wurden in «Suppen oder Salsen» oder in «den grünen Müsslein» gegeben, wenn schlechte Luft vorhanden war [1, S. 41]. Im Kräuterbuch von Otto Brunfels (1543) empfiehlt er, in Gebäckstücken Gundermann (Glechoma hederacea L.) als Mittel gegen «pestilentzisch vergyfft» mitzubacken [1, S. 42]. Die zusammen mit den Speisen gekochte Wurzel des Aronstabs (A. maculatum L.) wird vom kaiserlichen Leibarzt empfohlen. Die Pestilenzwurz/Geissrauten (Ruta capraria M.) wurde als Mittel gegen die Pest angepriesen, indem man sie mit dem Essen mitkochte oder ins Getränk gab. «Conservenzucker» mit Geissrautenkraut, mit Wurzeln der Meisterwurz (P. ostruthium W.D.J. Koch) oder mit Gewürznelken (S. aromaticum Merr. und L.M. Perry) wurde als Prophylaxe gegen die Pest empfohlen [1, S. 23, 60, 75, 91].

Mit Zucker angereicherter Sirup aus Heilpflanzenextrakten wurde prophylaktisch ebenfalls empfohlen. Schwarzwurzel (Scorzonera hispanica L.), Benediktenkraut (C. benedictus L.) und Sauerampfer (Rumex acetosa Campd.) wurden unter anderem als Zutaten für den Sirup empfohlen [1, S. 45, 127, 132].

\section{Medizinalwein}

Unter Medizinalwein verstand man dazumal einen mit Teilen bestimmter Heilpflanzen angereicherten Wein. Pflanzenkraut oder Pflanzenwurzeln wurden in Wein eingelegt, wodurch mit der Zeit ein Auszug entstand, oder sie wurden direkt mit Wein eingenommen. Der Wein wurde manchmal auch erhitzt. So gab es Küchenschellenwurzelwein (Pulsatilla vulgaris Mill.), d.h. «Vinum ex radicibus Pulsatillae», und Alantwein (Inula helenium L.), d.h. «vinum elunatum». Beifusswurzel (Artemisia vulgaris L.), Wurzel der stängellosen Eberwurz (Carlina acaulis L.), unreife Beeren des Weinstocks (Vitis vinifera L.) und Samen des Rittersporns (Delphinium staphisagria L.) wurden ebenfalls in Wein oder mit Wein eingenommen [1, S. 82, 87, 126, 131-133].

\section{Medizinalessig}

Auch Essig wurde als «Trägerstoff» für Heilpflanzen benutzt, teilweise kombiniert mit Theriak. Sowohl Tabernaemontanus als auch Matthiolus empfahlen in Essig eingelegten Knoblauch (A. sativum L.): «Wider die Pestilenz/ schneid Knoblauch in gutem scharfen Essig/trinck ihn warm/ist wohl so gut als manche theure Arzney.» Holderessig mit Engelwurz (A. archangelica L.) wurde von Paracelsus empfohlen.

Der Essig wurde sowohl kalt als auch warm eingenommen, manchmal nur tropfenweise, manchmal auch in grösseren Mengen [1, S. 40, 72].

\section{Schlussfolgerungen}

Der Kampf um das Überleben hatte die Menschen zur Zeit der Pest voll im Griff. Die Auflistung zahlreicher medizinischer Anwendungsformen der Heilpflanzen in dieser Arbeit zeigt den Willen der damaligen Bevölkerung, sich dieser grausigen Krankheit nicht ohne Widerstand zu ergeben.

«Helf Gott», «Zum Wohl» und «Gesundheit» sind Redewendungen in unserer Sprache, die aus der Zeit, als die Pest noch wütete, stammen [15]. Damit wünschte man seinen Mitmenschen Verschonung vor der fürchterlichen 
Krankheit. In der heutigen Zeit der hochentwickelten Medizin sind wir der Meinung, viele pathogene Prozesse im Griff zu haben. Die aktuelle Situation um die Verbreitung des H1N1-Virus (Mexikanische Grippe) lehrt uns aber anderes. Plötzlich kommen uns die einfachsten (Schutz-)Massnahmen aus der Zeit des Mittelalters sehr bekannt vor; ein Blick auf die Internetpräsenz des BAG genügt. In Essig getränkte Stofftücher, vor die Nase gehalten, machen Platz für Mundschütze. Das Vermeiden von Massenansammlungen wird heutzutage genauso empfohlen wie vor 550 Jahren. Quarantäne dient als Schutzmittel - damals wie heute.

«Während Rieux den Freudenschreien lauschte, die aus der Stadt aufstiegen, erinnerte er sich nämlich daran, dass diese Freude immer bedroht war. Denn er wusste, was dieser Menge in Freudentaumel unbekannt war und was man in Büchern lesen kann, dass nämlich der Pestbazillus nie stirbt und nie verschwindet, dass er jahrzehntelang in den Möbeln und in der Wäsche schlummern kann, dass er in Zimmern, Kellern, Koffern, Taschentüchern und Papieren geduldig wartet und dass vielleicht der Tag kommen würde, an dem die Pest zum Unglück und zur Belehrung der Menschen ihre Ratten wecken und zum Sterben in eine glückliche Stadt schicken würde» [16].

Pandemien wird es immer geben, Bakterien und Viren wird es immer geben, $Y$. pestis wird es immer geben. Sie gehören zu uns - wie wir zum Leben, wie wir zum Tod. Helf Gott - zum Wohl - Gesundheit!

\section{Dank}

Während meiner Ausbildung in Pflanzenheilkunde und Phytotherapie bin ich immer wieder auf die Betitelung «Pestpflanzen» gestossen. Als ich mich im Rahmen des CAS «Ethnobotanik und Ethnomedizin» mit der Pest als epidemische Krankheit auseinandergesetzt habe, wurde mir erst deutlich, wie grauenvoll diese Krankheit damals wirklich war und welche Folgen das Wüten der Pest auf den einzelnen Mensch und die Gesellschaft und deren soziale Systeme hatte. Diese schriftliche Arbeit ist all den Menschen gewidmet, die in der Vergangenheit den Kampf mit der Pest auf sich nehmen mussten, ohne die heutigen medikamentösen Möglichkeiten nutzen zu können.

\section{Disclosure Statement}

In Bezug auf diese Arbeit bestehen keine Interessenkonflikte.

Ellen Wulfers ist Kinderphysiotherapeutin und arbeitet in ihrer eigenen Praxis für Kinderphysiotherapie in Fislisbach (AG). Sie hat eine Ausbildung in Pflanzenheilkunde und Phytotherapie in Freiburg in Breisgau, Deutschland, absolviert. Anschliessend hat sie das CAS «Ethnobotanik und Ethnomedizin» an der Universität Zürich abgeschlossen, in dessen Rahmen diese Projektarbeit geschrieben wurde. Im Moment nimmt sie am CAS «Gartentherapie» an der ZHAW Wädenswil teil, das sie im Februar 2014 mit dem Thema «Stressbewältigung durch Naturelemente am Arbeitsplatz» abschliessen wird. Ihr Interessenfeld gilt dem Einfluss der Natur auf das Wohlbefinden des Menschen - früher wie heute.

\section{Literatur}

1 Müller KM: Pestpflanzen; Heilkräuter wider den schwarzen Tod. Freiburg, Lavori, 2005.

2 Dobson M: Seuchen, die die Welt veränderten. Hamburg, National Geographic, 2007.

3 Esser (Thilo): Pest, Heilsangst und Frömmigkeit. Studien zur religiösen Bewältigung der Pest am Ausgang des Mittelalters. Altenberge, Oros, 1999.

4 http://yersiniapestis.info/krankheit.html.

5 Hadem M: Die Pest im Mittelalter. München, GRIN, 2004.
6 http://de.wikipedia.org/wiki/ alexandrejeanyersin.

7 www.fsbio-hannover.de.

8 http://de.wikipedia.org/wiki/Yersinia pestis.

9 http://bag-admin.ch/ infektionskrankheitenvona-z/pest.

10 http://de.wikipedia.org/wiki/Pest.

11 Porta GB: Magiae naturalis. Frankfurt, Libri viginti, 1516, pp 659-660.

12 Brosse J: Magie der Pflanzen. Olten und Freiburg, Walter, 1992, p 171.
13 http://de.wikipedia.org/wiki/bisamapfel.

14 Fischer-Rizzi S: Botschaft an den Himmel. Aarau, AT, 2001

15 Meier M: Pest: die Geschichte eines Menschheitstraumas. Stuttgart, KlettCotta, 2005, p 76 .

16 Camus A: Die Pest. Reinbek, Rowohlt Taschenbuch, 1962. 